\title{
Vivencia de la sexualidad en el adulto mayor - Tacna 2016
}

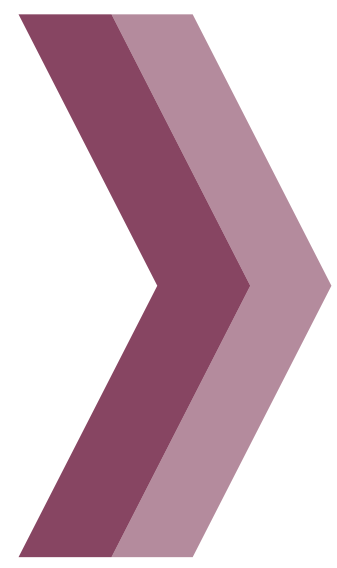

\section{Experience of sexuality in the elderly - Tacna 2016}

\section{Gema Natividad Sologuren ${ }^{1}$ Carmen Luisa Linares Torres ${ }^{1}$}

1. Doctora en Ciencias de la Educación, Universidad Nacional Jorge Basadre G. Escuela de Obstetricia. Facultad de Ciencias de la Salud

\section{RESUMEN}

INTRODUCCIÓN: La sexualidad en los adultos mayores es un tema, para muchos, desconocido, las personas creen que una vez cumplida la etapa reproductiva se acaba con ella toda expresión de afecto y sexualidad, considerándolos seres asexuados. El objetivo fue interpretar el significado que tiene la sexualidad para los adultos mayores. MATERIAL Y MÉTODOS: Es una investigación cualitativa, descriptiva. La unidad de observación fueron los adultos mayores hombres y mujeres de 60 a 71 años, que refirieron como estado civil: solteros, convivientes o casados. Se formaron tres grupos focales. RESULTADOS: Los adultos mayores están interesados en la sexualidad, son conscientes de sus limitantes para disfrutar su vida sexual como: disminuida fortaleza física y problemas de salud. Para las mujeres, mantener relaciones sexuales es parte de su necesidad emocional, condicionan su disfrute sexual al amor de su pareja, considerando un acto de entrega y afecto al hombre que aman, pueden mostrarle su cuerpo desnudo. Hay temor en los varones a no tener una respuesta sexual adecuada, consideran que pueden satisfacer a sus parejas no solo con la penetración sino también con caricias íntimas. Los varones tienen claro que sus parejas han disminuido su libido como producto de la menopausia, lo que limita su disfrute sexual. La mayoría no muestra predisposición a mejorar sus condiciones, no utilizan fantasías sexuales para lograr placer sexual. Tampoco tienen sueños eróticos que los ayuden a lograr mayor excitación para una mejor respuesta sexual. CONCLUSIÓN: Los adultos mayores tienen vivencias de su sexualidad con limitaciones propias de su edad.

PALABRAS CLAVE: Adultos mayores, sexualidad, vivencias

\section{ABSTRACT}

Sexuality in older adults is a topic, for many, unknown, people believe that once the reproductive stage is completed, all expression of affection and sexuality ends, considering them asexual beings. The objective of this research is to interpret the meaning of sexuality for older adults. It is qualitative, descriptive research. The observation unit was the elderly men and women from 60 to 71 years, who referred as civil status: single, cohabiting and married. Three focus groups were formed. RESULTS: Older adults are interested in sexuality, they are aware of their limitations to enjoy their sexual life as: their diminished physical strength and health problems. For women, having sex is part of their emotional need, they condition their sexual enjoyment on their partner's love, considering an act of devotion and affection to the man they love, they can show him their naked body. There is fear in men not to have an adequate sexual response, they believe that they can satisfy their partners not only with penetration but also with intimate caresses. Males are clear that their partners have decreased their libido as a result of menopause, which limits their sexual enjoyment. However, the vast majority shows no predisposition to improve their conditions using, for example, lubricants. They do not use sexual fantasies to achieve sexual pleasure. Nor do they have erotic dreams that help them achieve greater excitement for a better sexual response.

KEYWORDS: Seniorcitizens, sexuality, experiences 


\section{INTRODUCCIÓN}

El término sexualidad tiene muchos significados que dependerá entre otros aspectos, de las mismas personas, sus vivencias, la etapa de vida en que se encuentren, su educación. En la vejez el concepto de la sexualidad se basa principalmente en la "calidad de la relación" integrando componentes tales como: personalidad, intimidad, género, pensamientos, sentimientos, intereses y valores, etc (1).

Maslow, define la salud sexual Geriátrica, como "la expresión psicológica de emociones y compromisos que requiere la mayor cantidad y calidad de comunicación entre compañeros en una relación de confianza, amor, compartir y placer, con o sin coito" (2). La sexualidad en los adultos mayores sigue considerándose como un tabú, llena de prejuicios. Se piensa que ya perdieron todo interés por lo sexual y que si aún mantienen relaciones sexuales, se les califica como pervertidos. Muchos desconocen aún que el potencial sexual puede mantenerse hasta la muerte, con las limitaciones propias del envejecimiento. Consideramos que el ejercicio de la sexualidad en el adulto mayor constituye una expresión (física, afectiva) propia de todo ser humano que contribuye a una mejor calidad de vida y autoestima.Planteando como objetivo interpretar el significado que tiene la sexualidad para los adultos mayores.

\section{MATERIAL Y MÉTODOS}

Se trata de una investigación cualitativa, descriptiva, enmarcada dentro del área de las ciencias de la salud y la psicología, siendo su unidad de observación adultos mayores hombres y mujeres, cuyas edades fluctuaron entre los 60 a 71 años de edad quienes refirieron como estado civil: solteros, convivientes, casados. Se formaron tres grupos focales. Se aplicó una guía conversatorio.

\section{RESULTADOS}

\section{ACTITUDES, PREJUICIOS Y CONDUCTAS DE LOS ADULTOS MAYORES (HOMBRES Y MUJERES) FRENTE ALTEMA DE LASEXUALIDAD}

La sexualidad para la mayoría de los adultos mayores es un tema, aún complejo difícil de tratar abiertamente, considerándola en menor medida un tabú.
"Yo nunca he recibido información sexual porque no había, se avergonzaban de hablar de esas cosas". Mariano.

"Nunca he recibido información, antes no había eso... No había... Ni enamorado, todo era a escondidas......ahora pues, en la puerta estas con tu chico bien parado al frente de tu mamá, antes no había eso... Mi papá me sabe decir: iHay una soga grande en el techo, ahí te voy a colgar! ..... me decía a la hora hay que llegar, si tenías un chico y te encontraban, tenías que ir a tu casa corriendo.........así era antes, ahora ya no pues". Sabina

Los adultos mayores que cuentan con educación superior, muestran una actitud más positiva hacia la sexualidad, pero con una visión fragmentada, orientada a la reproducción.

"Sexualidad ...si, cuando estuve estudiando superior en la universidad, pero no tan profundo, solamente nos enseñaban lo principal o cosas puntuales como los métodos que uno debe aplicar: el método del ritmo o cuidarse con preservativos, ese tipo de información". Marcos

\section{FORMAS EN QUE LOS ADULTOS MAYORES EXPRESAN SUS SENTIMIENTOS, EMOCIONES $Y$ TEMORES FRENTE AL TEMA DE LA SEXUALIDAD.}

No cuentan con un espacio en el que puedan dialogar, expresar sus inquietudes, para poder aprender sobre cómo vivir su sexualidad en esta etapa de vida. Sienten que sus hijos no tienen interés por conversar sobre sexualidad con ellos, que son pocas las ocasiones y las personas con las que pueden hablar un poco del tema, y son amigos o amigas con las que en algunas oportunidades lo hacen.

"Sobre sexo, muy poco porque....no sabría cómo decirles porque... uno ya no conversa mucho de esas cosas". María

"Muy poco...uhmm... con mi pareja si, si tenemos esa comunicación y hablamos, pero no tanto, pero siempre conversamos en casa con ella cuando descansamos, más que todo en la noche y en el día uno tiene que salir a trabajar así que casi no! no tenemos tiempo necesario como para conversar en el día, pero en la noche si conversamos,.. Pero no lo he hecho con mis hijos". Marcos

Algunos adultos mayores con instrucción superior 
muestran más interés por expresar sus necesidades frente a los cambios biológicos que sufren sus parejas como producto de su edad.

"Si, de todo un poco ah... digamos sobre poses, sobre el uso de condones, de un montón de cosas hablamos, de las pastillas, de los lubricantes, porque ya cuando la persona, la mujer más que todo... este... ya pasó la menopausia sus genitales ya no lubrican como cuando era joven, entonces para ella el acto sexual es muy doloroso, y yo respeto esas cosas a pesar de que existen lubricantes mi esposa ino quiere usar!, bueno yo respeto pues si no quiere usar es que no quiere sexo, la respeto, tampoco soy un chiquillo que me desespero portenersexo". Carlos

Para algunos adultos mayores, el encuentro sexual lo valoran en relación a la afectividad para que sea satisfactorio. Para expresar su sexualidad, con sus parejas, necesitan mostrar ciertas condiciones, tales como la comunicación, respeto, amor, y comprensión

"Para mí, lo básico y fundamental en todo, es la comunicación, nunca se va a llegar a nada sino hay una buena comunicación, eso disipa las dudas, aclara problemas, resuelve todo". Yolanda.

"El amor, la comprensión mutua de pareja y sobre todo bastante amor, yo creo que cuando hay amor todo lo demás es secundario. La comprensión y entender a la pareja. Debe haber diálogo". Víctor

Asimismo, consideran que importante la parte física, caricias, tocamientos a su pareja, en especial en los genitales. De esta forma asocian la vivencia de una relación sexual al logro del placer sexual.

"uhmm... las caricias, si existe amor todo va bien. En la sexualidad hay más que besos, hay caricias o tocamientos en uhm... con la pareja en las partes intimas de uno, vas descubriendo poco a poco con besos, con la mano o con los labios lo que uno normal hace con una pareja, entre una mujer y un varón". Marcos

\section{SIGNIFICADO DEL PLACER SEXUAL SEGÚN SEXO EN LOS ADULTOS MAYORES.}

Afirman que es "normal" tener interés sexual a su edad y este interés es en ambos sexos.

"Las personas de más de 60 años tienen interés sexual... yo creo que sí, mi opinión que sí.". Anónimo
"Con mi esposo ambos tenemos interés sexual todavía, aunque no tan frecuentemente, antes sí". Carola

Algunos adultos mayores consideran que tener más de 60 años no inhabilita a la persona para tener prácticas sexuales. Sin embargo, consideran que tienen suficientes condiciones para tener vivencias sexuales y que por ello tienen interés por los encuentros sexuales.

"Y porque no?jajaja...todas las personas tienen diferentes intereses, tener 60 o más no inhabilita a las personas a hacer las cosas que quieran y si quieren hacer "eso" que lo hagan normal jajajajaja..." Yolanda

"Bueno en lo que a mírespecta, yo sí... Digamos cuando eres joven y miras una chica, hasta podría tener una erección en ese momento, lo que ya no sucede cuando eres adulto, puedes mirar un par de hermosas piernas pero no vas a tener erección, necesitas más estimulación ya sea con el tacto, con besos, abrazos, palabras". Carlos

Para los adultos mayores el interés sexual está asociado al amor, y el amor de pareja puede durar hasta esta etapa de la vida.

"Yo creo que si hay interés sexual porque en una pareja el amor nunca muere, solamente cuando uno deja de existir como ser humano, morirá no, pero mientras tanto el amor sigue latiendo, sigue en la pareja, si uno es casado tiene que respetar siempre a su pareja y... el amor es lo más sublime que le pudiera pasar al ser humano, comprenderse mutuamente y entregarse intimamente a su pareja". Marcos

Los adultos mayores consideran que el interés sexual es de ambos sexos en la vejez.

"Tanto hombre como mujer...yo creo... más en el hombre porque quiere estar con su mujer...". María

Aseguran, que las personas en esta etapa de la vida tienen atractivo sexual y lo relacionan a sus rasgos de personalidad agradable, considerando que el amor por la pareja pueda disminuir o desparecer con la convivencia y el tiempo.

"Sí, porque me siento atraída por mi pareja". Teresa "Una persona de la tercera edad, tiene atractivo iclaro! uno se siente joven y hábil todavía...". Anónimo

Los adultos mayores relacionan el atractivo sexual con la fortaleza física. 
"Depende de la fortaleza física y la salud ehh...creo que ahí manda el organismo, atractivo... creo que siempre cada persona tiene su atractivo, no creo que esa atracción se pierda al llegar a esta edad, también las personas adultas tenemos nuestro atractivo". Víctor

Si bien el interés sexual de los adultos mayores no desaparece luego de los 60 años de edad, esta vivencia es menos intensa.

"Tienen interés sexual pero no tan seguido. El hombre llega cansado quiere dormir... la mujer está cansada quiere dormir, pero de vez en cuando siempre pues señorita, usted sabe". María

"Claro que sí, pero por ejemplo a mi esposa no, no soy cruel, pero ella no te incentiva para nada, por lo mismo que perdió el interés en el sexo, yo si tengo atractivo, yo sé pues, hay otras mujeres que si quieren estar conmigo a veces, si a veces, jajaja". Carlos

En la tercera edad, el cuerpo está íntimamente relacionado con la vivencia de la sexualidad, el placer y el disfrute. Los adultos mayores perciben que el cuerpo desnudo les permite tener encuentros sexuales satisfactorios. Por ello, no tienen temor de mostrarse desnudos ante su pareja.

\section{"Claro es importante ver el cuerpo". Virginia}

"Yo, con ella tenemos cierta confianza para vernos desnudos". Anónimo

Para las mujeres la sexualidad está vinculada a su intimidad y al amor hacia su pareja, ello les da la seguridad de mostrar su cuerpo desnudo a su pareja sin temores.

"Sí, me muestro noma desnuda, no tengo vergüenza. No, no hay vergüenza". Carola

"No, en absoluto. Yo tengo confianza con mi pareja, y si a ella le gusta observarme, isí!, me desnudo, me saco mi ropa. Yo duermo así desnudo, semidesnudo, me pongo, solamente una polerita y listo". Marcos

Los varones adultos mayores se muestran bastante desinhibidos, predispuestos, a mostrar su cuerpo desnudo a la pareja durante un encuentro sexual. Fundamentan su percepción en el hecho que han vivido junto a su pareja por muchos años, y han ido envejeciendo, por tanto, ambos conocen sus cuerpos y tienen confianza de mostrarlo al otro.
"Ya es conocido el cuerpo, normal, normal estoy calato, cuando son jóvenes tienen vergüenza tanto la mujer como el hombre, cuando el hombre conoce el cuerpo de la mujer ya no hay vergüenza de nada, ya no importa. Entre adultos así viejitos ya no hay vergüenza". Máximo

\section{EFECTOS DEL ENVEJECIMIENTO EN EL EJERCICIO DE LA SEXUALIDAD EN LOS ADULTOS MAYORES.}

Como producto del envejecimiento, los adultos mayores han disminuido la frecuencia de sus encuentros sexuales.

"Bueno, el fin de semana por decir, porque otros días uno está laborando, trabajando y el fin de semana uno descansa y en ese lapso de descanso uno tiene relaciones con su pareja". Marcos

"Para estar, por ejemplo hay que satisfacer realmente a la pareja, no es sólo sexo, por ejemplo lo que le gusta a la mujer, darle ánimo para trabajar, hacerla reír, un beso, una caricia... puede ser que te de ganas, si no tienes ganas: no hay". Máximo

"No tengo relaciones muy seguido. Pero si cuando mi marido quiere estar conmigo, no le puedo decir que no, a la semana: 2 veces por semana". María

Los adultos mayores tienen interés por mantener una vida sexual activa, pero admiten que sus cuerpos se sienten cansados y no tienen la fortaleza física suficiente para aumentar su frecuencia; su libido ha disminuido, tienen problemas para tener erecciones, surgiendo el temor a que sus parejas le sean infieles. Se cansan con facilidad, tienen sueño. Algunos refieren que el placer lo obtienen con otras demostraciones afectivas, como besos, caricias, paseos y detalles cariñosos. Los adultos mayores explican que la baja frecuencia de los encuentros sexuales, está determinada por el proceso de envejecimiento.

"Eso es una vez al mes, como digo ya no hay ganas y duermes como un bebe tranquilo...". Máximo

"jajajaja... no, como le dije no es prioridad para mí, creo que estoy en otra etapa en la que el cariño, el amor se puede demostrar de tantas maneras, que el sexo no es lo básico para que una relación funcione. Lo mismo que te dije anteriormente, hay tantas maneras, no solo el sexo: las caricias, los besos, son las acciones que están presentes en mi vida y a esto lo complementan los detalles, los paseos, esos son los momentos más significativos para mi". Yolanda 
La mayoría de los adultos mayores consideran necesaria la penetración para la satisfacción sexual. Son breves y categóricos al afirmar que esta es la que determina sus encuentros sexuales.

"La penetración... él ya está viejo, no es lo mismo, pero igual, ahora como uno está viejo... ya no es tanto, hay que complacer al marido, hasta donde se pueda..." Anónima

"Es la parte más importante para mí en el acto sexual, claro que hay otros aspectos pero para mí, la penetración es importante". Víctor

Algunos adultos mayores consideran que la penetración no es tan importante para tener placer sexual. Mencionan que puede bastar con besos y caricias en sus genitales.

"Uhmm no... veo más... uhmm... las caricias, los besos". Carola

"No tanto, es secundario. Cuando existe amor, no es tan importante la penetración, uno puede jugar con su pareja, buscando con besos, con sus labios o con sus manos en partes íntimas de la pareja y no llegar a la penetración, inclusive puedes descansar normal. Pero si es necesario, si no hay ningún inconveniente, pero no es tan preferencial". Marcos

Respecto a las prácticas sexuales realizadas por los hombres adultos mayores, está la masturbación.

"Caricias... con las manos, caricias con las manos...si recurro ciertas veces a estimularme yo mismo... de vez en cuando, señorita..., yo también llego a masturbarme en mis momentos...". Anónimo

\section{"Caricias con mano y coito". Teresa}

Los adultos mayores consideran que la estimulación con besos en los labios es muy importante para iniciar un encuentro sexual.

"Caricias con las manos, con los labios, antes de tener el acto sexual. Es algo normal". Yessi

"Realmente uno busca en la mujer su debilidad con caricias y besos cuando la encuentras, automáticamente pide la mujer relación, si ya has buscado a la mujer tienes que buscar sus caricias, su boca, besos en el cuello, en los senos por ejemplo, cuando encontraste su debilidad... la mujer también te agarra jajajajaja... ahí y ya... también la mujer te busca. Si, con tu pareja puedes hacer estas cosas, con otra persona no, no es como la mujer que ya has conocido". Máximo

Los adultos mayores afirman que se encuentran satisfechos en sus encuentros sexuales. En realidad, se puede percibir un conformismo respecto a sus expectativas de satisfacción sexual.

"Yo estoy contenta, satisfecha porque... ya para mí, no es lo mismo, porque a mi edad más me dedico a descansar, estar con los nietos. Uno por cumplir al marido ya". María

"Para conseguir mayor satisfacción, hay que tener confianza dialogar más que todo con ella". Anónimo

\section{DISCUSIÓN}

Herrera, A (1), Santiago-Chile, en el año 2003, sostiene que el comportamiento sexual en la vejez depende de muchos factores: salud en general, disponibilidad de un compañero (a) sano (a), personalidad, actitudes hacia y de los otros, nivel de educación, nivel social, creencias sexuales, actitud sexual previa, intereses y prácticas previas, grado de satisfacción con la vida, etc. En el mismo sentido, Cornachione, M, (3) Córdova, el 2006, plantea que, cuando las personas envejecen cambia la estructura corporal y su funcionamiento. Los cambios físicos que se producen con el envejecimiento son distintos en el hombre y en la mujer. Si bien es cierto que la frecuencia de las relaciones sexuales se disminuye, esta reducción guarda directa relación con los hábitos sexuales que hombres y mujeres han tenido durante la juventud y la adultez.

Weeks, J. (4), México, 1998, afirma que el hombre que desarrolla una vida sexual activa y no pierde la confianza en sí mismo será capaz de lograr erecciones y orgasmos hasta edades muy maduras. Desde el punto de vista fisiológico, sabemos que en el adulto mayor los niveles de testosterona, si bien bajan, lo hacen a un nivel que permite un buen desempeño sexual.En esta investigación se encuentra que los adultos mayores relacionan las carencias en su vida sexual con el proceso de envejecimiento natural que sus cuerpos han sufrido. Los varones consideran que su vida sexual depende de la disponibilidad de su pareja, fundamentalmente; $y$ en el caso de las mujeres, de las actitudes de su pareja como la demostración de los afectos, directamente relacionados con el amor y el compañerismo. 
La sexualidad en el hombre y la mujer añosos sufre modificaciones que son generadas por el propio envejecimiento, y exigen una adaptación de la conducta sexual de la pareja de ancianos que propicie una sexualidad gratificante, libre de frustraciones y ansiedades que podrían conducir a la interrupción innecesaria de la actividad sexual. Aunque ambos sexos pueden conservar su capacidad sexual hasta edades muy avanzadas, el hombre suele ser más vulnerable que la mujer. Kaplan, $\mathrm{H}(5)$, Washington, en el 2000 , sostiene que en el adulto mayor, aumenta la necesidad de estimulación, se reduce la respuesta visual y táctil respecto a los hombres en etapas previas; disminuye la rapidez de la erección y esta es menos firme. En este sentido, encontramos que los adultos mayores, de esta investigación presentan un descenso en la frecuencia de los encuentros sexuales, por un descenso en la libido, presentando disfunción eréctil. En el caso de las mujeres, Dolto, F, (6) Barcelona, en 1999, menciona, que uno de los problemas más notorios es la escasa o nula lubricación de la vagina que ocasiona dispareunia. Estos datos corroboran los encontrados en esta investigación, que mencionan que resequedad de la vagina, y falta de lubricación les impide disfrutar sus encuentros sexuales, lo cual genera preocupación por los efectos de la menopausia.Existe una inquietante tendencia a equiparar la actividad sexual con la coital,cuando el encuentro sexual, Abarca variadas actividades o formas de relación entre dos personas y NO SE LIMITA SOLO al coito o penetración, que provoque excitación sexual con o sin orgasmo: abrazos, masajes, caricias o besos en el cuerpo, zona genital, sexo oral, penetración (con o sin eyaculación dentro de la vagina), sexo anal. Flores Colombino, A (7) Montevideo, en 1989, afirma, que la necesidad de relacionarse con otras personas, de expresar sentimientos, de recibir afecto de la otra persona no se pierde por más viejos que sean los individuos. Es en ese sentido, encontramos en nuestra investigación, dos posturas significativas, por un lado están los adultos mayores que consideran necesaria la penetración para la satisfacción sexual; y por otro lado, aquellos que dan un gran peso a otras demostraciones afectivas como besos y caricias y otros detalles cariñosos.

Kinsey y otros (citado por Herrera, 2003) (1), demostraron que la masturbación es bastante común entre los ancianos (levemente menor que en los jóvenes). En el presente estudio fueron los varones los que reconocieron masturbarse para obtener placer sexual. Las mujeres se muestran tímidas para ahondar en el tema. Sin embargo, reconocen que les agradan las caricias que les brindan sus parejas en el área genital.

Corominas, García, Ortega y Serna (8) Madrid, en 1995, en su investigación con ancianos sobre su vida sexual, determinan que un $74 \%$ de mujeres no tienen sueños eróticos y el $44 \%$ de hombres que sí los tienen; además, la mayoría de las personas que han contestado afirmativamente señalan que alcanzan el orgasmo. En la presente investigación, se encontró similar información ya que la mayoría de los adultos mayores entrevistados manifiesta no tener sueños eróticos. Los autores antes mencionados, también señalan que el $84 \%$ de los varones y el $89 \%$ de las mujeres manifiestan no tener ningún tipo de fantasía sexual cuando realizan el acto o se autoestimulan; de los 32 que contestan afirmativamente, el mayor porcentaje corresponde a los casados seguido de los viudos. En la presente investigación solo uno de los entrevistados admite que a veces se masturba para obtener placer sexual, y es varón. En el presente estudio se puede notar que para muchos/as adultos mayores es muy difícil hablar de su sexualidad. Es un tema evasivo para ellos porque ellos mismos lo admiten no han tenido información sobre sexualidad durante su adolescencia y juventud.

La información recibida fue básicamente sobre anticoncepción, en el mejor de los casos. Para, Juger, C (9) Santiago, en el 2010, en un estudio sobre la sexualidad en los adultos mayores, concluyó que los adultos mayores son un grupo de hombres y mujeres nacidos en las dos o tres primeras décadas, por lo tanto se comprenderá que una actitud ante la sexualidad aprendida en un contexto cultural de prohibición, que por otra parte choca radicalmente con los valores propuestos en la sociedad consumista actual, va a ir generando ideas negativas hacia las prácticas sexuales que se den con una perspectiva de comunicacióndiversión, que probablemente este factor sea uno de los que más contribuya a mantener una situación de negación de las necesidades afectivo-sexuales.

Finalmente, se puede concluir que, los adultos mayores están interesados en la sexualidad pero son conscientes de sus limitantes para disfrutar de su vida sexual. Estas limitantes son básicamente su disminuida fortaleza física y sus problemas de salud propios de la edad.Para las mujeres adultas mayores el mantener relaciones sexuales es parte de su necesidad emocional. Condicionan su disfrute sexual al amor de su pareja. Lo consideran un acto de entrega del cuerpo y afecto al hombre al que aman y confían, por lo que pueden mostrarle su cuerpo desnudo. Es frecuente el 
temor de los varones a no poder tener una respuesta sexual adecuada, sin embargo, consideran que pueden satisfacer a sus parejas no solo con la penetración sino también con caricias íntimas.Es más visible el envejecimiento de la mujer que el de los varones adultos mayores. Los varones tienen claro que sus parejas han disminuido su libido como producto de la menopausia, lo que saben limita su disfrute sexual. Sin embargo la gran mayoría de ellos no muestra predisposición a mejorar sus condiciones a través del uso, por ejemplo, de productos como lubricantes de genitales. Los adultos mayores no utilizan fantasías sexuales para lograr placer sexual. Tampoco tienen sueños eróticos que los ayuden a lograr una mayor excitación para poder tener una mejor respuesta sexual.

En consecuencia, consideramos recomendable que, el Gobierno Regional y Gobierno Local elaboren y ejecuten políticas públicas de preparación de hombres y mujeres para ésta etapa de la vida que se debe iniciar en la niñez y mantenerse en el tiempo.

\section{REFERENCIAS BIBLIOGRÁFICAS}

1. Herrera A. revista chilena de obstetricia y ginecología. Sexualidad en la vejez ¿Mito o realidad? Santiago, Chile 2003. págs., 150-162.

2. Maslow. H. Motivación y personalidad. Sagitario, 1954

3. Cornachione, M. Psicología del desarrollo. Córdoba: Editorial Brujas,(2006).

4. Weeks J. Sexualidad. México DF: Editorial Paidós Mexicana; 1998

5. Kaplan H, Sadock BJ. Sinopsis de psiquiatría. 8a edición. Washington DC: Editorial Médica Panamericana; 2000

6. Dolto F. Sexualidad femenina. Barcelona: Editorial Paidós Ibérica; 1999.
7. Flores Colombino A. Sexualidad en la tercera edad. 2. ed. Punto Láser P-8 Montevideo, 1989.

8. Corominas, C., García, M., Ortega, M. y Serna; C. Sexualidad y tercera edad. Revista de Enfermería 1995; 5: 6-13. Universidad Complutense. Disponible en: http://cisne.sim.ucm.es/record=b1770121

9. Juger C. Estudio sobre la sexualidad en los adultos mayores. Tesis para optar al grado de Licenciado en psicología. Universidad Academia de Humanismo cristiano. Santiago.(2010). Disponible en:

http://bibliotecadigital.academia.cl/bitstream/handle/123 456789/2277/tpsico348. pdf?sequence $=1$ \&isAllowed $=y$

\section{Correspondencia}

Gema Natividad Sologuren

gemasologuren5@hotmail.com
Fecha de recepción: 19 de mayo de 2017

Fecha de aceptación: 20 de junio de 2017 